\title{
DISCAPACIDAD INTELECTUAL Y CALIDAD DE VIDA ESCALA DE EVALUACIÓN
}

\section{INTELLECTUAL DISABILITY AND QUALITY OF LIFE EVALUATION SCALE}

Gerardo Alberto Moreno Medina gmoreno@jdc.edu.co

Fundación Universitaria Juan de Castellanos

Boyacá-Colombia

Ennyth Julyeth Alvarez Chaparro ${ }^{2}$ ejalvarez@jdc.edu.co Fundación Universitaria Juan de Castellanos Boyacá-Colombia

Fotografia de portada y Diagramación Sindy Catherine Charcas Ibarra 


\section{RESUMEN}

Objetivo. Evaluar la calidad de vida en personas con discapacidad Intelectual.

Diseño. Cualitativo de tipo fenomenológico.

Metodología. La población del centro de educación especial de Comfaboy comprende 70 Niños y jóvenes en condición de discapacidad intelectual, la muestra seleccionada a través de muestreo no probabilístico por conveniencia son dos personas. Se aplicó la escala INICO-FEAPS validada en Colombia que permite describir por medio de 72 ítems distribuidos en ocho dimensiones de calidad de vida, según el modelo de Schalock y Verdugo, se utilizaron dos cuestionarios de la escala, el autoinforme y el informe de otras personas

Resultados. En el análisis por dimensiones de la escala de calidad de vida, se destaca que las 2 personas evaluadas presentaron diversas puntuaciones, tanto en el autoinforme como en el informe de otras personas.

Conclusión. Los evaluados mostraron menores puntajes en las dimensiones de inclusión social y derechos, revelando la necesidad de orientar a las familias en la compresión de sus derechos y que estos le permitan ser parte de una sociedad incluyente.

Palabras clave: INICO-FEAPS, evaluación de la discapacidad, adultos, calidad de vida, discapacidad intelectual.

\section{ABSTRACT}

Objective. Evaluate the quality of life in people with intellectual disabilities.

Design. Qualitative nature, with a phenomenological type design.

Methodology. The population of the special education center of Comfaboy includes 70 children and young people in intellectual disability, the sample selected through non-probabilistic sampling for convenience are two people. The INICO-FEAPS scale validated in Colombia was applied, which allows the description of 72 items distributed in eight quality of life dimensions, according to the model of Schalock and Verdugo, using two questionnaires of the scale, the self-report and the report of other people.

Results. The analysis by dimensions of the quality of life scale, it is highlighted that the 2 people evaluated presented different scores, both in the self-report and in the report of other people.

Conclusion. Those evaluated showed lower scores in the dimensions of social inclusion and rights, revealing the need to guide families in understanding their rights and allowing them to be part of an inclusive society.

Keywords: INICO-FEAPS, disability assessment, adults, life quality, intellectual disability

\footnotetext{
Gerardo Alberto Moreno. gmoreno@jdc.edu.co

2 Ennyth Julieth Alvarez Chaparro ejalvarez@jdc.edu.co
} 


\section{INTRODUCCIÓN}

En la actualidad la definición de discapacidad intelectual se entiende como la presencia que coexiste con deficiencias cognitivas y en las funciones adaptativas del sujeto (Schalock et al., 20l0), por esta razón la evaluación de la calidad de vida en personas con discapacidad intelectual en el presente estudio es de vital importancia para comprender desde diferentes puntos de vista la subjetividad en el concepto de calidad de vida, y las dimensiones en las que sea susceptible la sugerencia por parte del evaluador para potenciar y/o mejorar aspectos que se perciban inferiores en su apreciación.

Osorio (2016) señala que los problemas afines a la discapacidad intelectual, tienen que ver sólo con la persona, sin tener en cuenta el entorno que lo favorece y que puede facilitarle o entorpecer su accionar en las diferentes situaciones o exigencias que se le proyectan.

Se cuenta también con la idea de concebir la discapacidad no sólo en la persona sino en el apoyo o las limitaciones que provee su entorno familiar, por tal motivo el instrumento para medir la calidad de vida es acertado ya que busca identificar rasgos característicos tanto en la persona con discapacidad intelectual como en sus cuidadores, tutores o familiares.

La valoración de la calidad de vida en familias con uno o más miembros en condición de discapacidad, es el tema central de los estudios interculturales, al determinar que su análisis no debería realizarse bajo una aproximación psicopatológica, sino más bien a través de una concepción en la que se prioricen las potencialidades y las capacidades del nucleo familiar (Verdugo, Córdoba y Gómez, 2006):

Existen investigaciones como la de Nussbaum, Sen, y Reyes Mazzoni (1996) que nos hace entender la calidad de vida como uno de los pilares de la evolución del ser humano en general, partiendo de esta concepción y tratando de aplicar la inclusión social de la mejor manera posible también para esta investigación se le da a la calidad de vida un papel preponderante necesario en la consolidación del ser humano independiente de su condición.

La expresión corporal, la actividad física y en general las actividades que permiten la motricidad y el movimiento juegan una parte importante en el desempeño personal y a su vez en la calidad de vida (Torres, 2013), en este sentido las dimensiones de bienestar físico y relaciones interpersonales evaluadas en la presente investigación en caso de encontrar debilidades pueden ser intervenidas a través de este tipo de actividades.

La legislación colombiana en torno a la discapacidad se encuentra prácticamente impresa en un papel, pero poco efectiva, un estudio realizado por Martínez-Rozo, UribeRodríguez, y Velázquez-González, 2015) demostró que, a pesar de encontrarse amparados por múltiples organismos y régimen aprobados y sustentados por el poder legislativo del país, la percepción de la población en condición de discapacidad es precaria frente a temas como educación, salud, empleo y transporte que son aportes para la construcción del concepto calidad de vida.

Según el Censo Poblacional DANE, realizado en 2005, en el departamento de Boyacá existe una prevalencia de discapacidad del 8,7 \%, refiriendo a 104087 personas en esta condición, porcentaje por encima del nivel nacional, que es de 6,3 \%. (Cobo Mejia, Sandoval Cuellar y Alvarado Rojas, 20I2).

Por lo anterior evaluar la calidad de vida de dos personas en condición de discapacidad intelectual del centro de educación especial Comfaboy-Tunja, permitió describir dimensiones como: inclusión social, autodeterminación, bienestar emocional, bienestar físico, bienestar material, derechos, desarrollo personal y relaciones interpersonales; a través de la valoración y estimación del autoinforme y el informe de otros. Para tal efecto el presente estudio aplico la escala INICO-FEAPS adaptada a la población colombiana, con la intención de abarcar en diferentes dimensiones los aspectos relevantes percibidos desde la persona en condición de discapacidad y sus tutores. (Henao-Lema, Verdugo-Alonso, y Córdoba-Andrade, 2015).

\section{MARCO CONCEPTUAL}

Durante los años 80, el término Calidad de Vida se adoptó como concepto sensibilizador que podía ofrecer a los profesionales de distintas disciplinas un lenguaje común y guiar las prácticas de los servicios humanos, más orientados ahora hacia la persona, su autodeterminación y el logro de una mayor satisfacción con su vida. A lo largo de los 90, las preocupaciones en torno a la conceptualización y evaluación del concepto tuvieron un mayor carácter metodológico. Superadas estas inquietudes, el siglo $X X I$ se presenta como aquél en el que el término Calidad de Vida no sólo teñirá las intenciones y acciones de individuos que gozan cada vez de mayores posibilidades de elección y decisión y optan por una vida de mayor calidad, sino también las de los servicios humanos en general, que se verán obligados a adoptar técnicas de mejora de sus procedimientos, en la medida que existirá un grupo de evaluadores que analizará sus resultados desde criterios de excelencia como es el de Calidad de Vida. (Cuervo Rodríguez 2014)

según censo poblacional del DANE, realizado en el departamento de Boyacá, existe una prevalencia de discapacidad del 8,7 \%, refiriendo a 104087 personas en esta condición, porcentaje por encima del nivel nacional, que es de 6,3 \%. (Cobo, Sandoval 2012)

La escala INICO- FEAPS adaptada a la población colombiana, cuenta con baremos para las dos versiones (autoinforme e informe de otras personas) que permiten transformar las puntuaciones compuestas (suma de las puntuaciones directas obtenidas en los ítems de cada dimensión) en puntuaciones estándar $(M=10 ; D T=3)$ y percentiles. Asimismo, se puede calcular una puntuación total, denominada índice de calidad de vida $(M=100$; DT $=15)$. Finalmente, las puntuaciones estandarizadas se representaron de forma gráfica en un perfil de calidad de vida que facilita la interpretación de los datos y el análisis de discrepancias y 
Discapacidad intelectual y calidad de vida escala de evaluación

semejanzas entre las dos evaluaciones. (Verdugo Alonso et al. 2013).

\section{Método}

La presente investigación de carácter cualitativo que se enfoca en comprender y entender los fenómenos y además explorar desde la perspectiva de los participantes en ambientes naturales relacionados con su contexto, con un diseño de tipo fenomenológico que busca entender las experiencias de personas sobre un fenómeno o múltiples perspectivas de éste y explorar las categorías: calidad de vida y discapacidad intelectual.

\section{Participantes:}

La población del centro de educación especial de Comfaboy comprende 117 Niños y jóvenes en condición de discapacidad, física, sensorial y psíquica; la directora del programa manifiesta que el $70 \%$ de la población presenta como diagnóstico de base la discapacidad intelectual, y un nivel socioeconómico I y 2 del régimen subsidiado, la muestra seleccionada a través de muestreo no probabilístico por conveniencia son dos personas en condición de discapacidad intelectual del programa. (Otzen \& Manterola,2017)

Para el presente estudio, se cuenta con dos usuarios del programa de educación especial O.R.H.M de 34 años género masculino con Diagnostico Síndrome de Down asiste al programa desde hace $2 \mathrm{I}$ años con una interrupción de 5 años, tiene prueba de coeficiente intelectual con resultado de 45 , es sociable, dinámico y activo, se desempaña fácilmente en los desplazamientos, su familia es de gran apoyo en el proceso, en el ámbito de lecto-escritura los avances son lentos debido a su C. I; conoce cantidades y sabe manejar dinero tal como lo refiere la psicopedagoga de la institución; de la misma manera A.F.F. $M$ es el segundo paciente con 19 años género masculino ingresa al instituto por dificultades en la marcha $y$ postura, posteriormente a través de los exámenes realizados se halla por medio de la prueba de C.I que arroja 55 puntos, se fortalece proceso de lectura que aunque lee bien pero en escritura presenta dificultades en separación de palabras, silabas inversas y las combinaciones, en matemáticas presenta mayor dificultad en sumas y restas, a nivel social A.F.F.M se muestra atento y colaborador tanto con profesores como estudiantes, muy sociable, practica danza y deportes, es un guía natural apoyado de forma permanente por la familia al generar independencia.

\section{Instrumentos}

Se aplicará la Escala INICO-FEAPS adaptada y validada en población colombiana. (Henao-Lema, Verdugo- Alonso, Córdoba- Andrade, 2015)

La escala consta de dos formas paralelas cada una con 72 ítems distribuidos en las ocho dimensiones de calidad de vida del modelo de Schalock y Verdugo (2003). Las dos formas hacen referencia a: el informe de otras personas que diligencia un tercero que conozca bien a la persona y el autoinforme que debe ser contestado por la persona con discapacidad intelectual a través de un entrevistador o de forma autónoma con apoyo de otra persona que garantice la adecuada comprensión de los ítems y del formato de respuesta. Todas las preguntas tienen un formato de respuesta tipo Likert con cuatro opciones: nunca, algunas veces, frecuentemente y siempre.

\section{Procedimiento}

Para la presente investigación se realizará la aplicación de la escala de medición de calidad de vida INICO-FEAPS; la escala se aplica en dos etapas, inicialmente un autoinforme con un tiempo estimado de 45 minutos de duración y se realiza con la persona con discapacidad intelectual; la segunda parte consiste en un Informe de más o menos 15 minutos donde otras personas que pueden ser: profesionales, familiares, amigos, allegados que conozcan muy bien a la persona con Discapacidad Intelectual que en este caso corresponde a los participantes en la investigación; se analizará la información recolectada para cada uno y se compara con la aportada por su tutor, de esta manera "la escala busca identificar el perfil de la población a partir de la evaluación de aspectos observables de su calidad de vida, en la que se contrastan la propia perspectiva con la de otros que lo conozcan bien.

Esta información se constituye en un referente para el diseño, ejecución y seguimiento de los progresos y resultados de diferentes intervenciones dirigidas a la población." (Schalock, Alonso, \& Ángel, 2007)

Como punto de partida se aplica la escala INICOFEAPS, junto a todos los instrumentos diseñados para tal fin. Posteriormente, corresponde analizar la percepción desde los dos puntos de vista: autoinforme e informe de otras personas, con el fin de encontrar la dimensión que se encuentre reducida según la escala.

\section{Resultados}

Según la organización mundial de la salud (20II), en la actualidad existe una evolución de la dinámica en la caracterización de la discapacidad. En este sentido numerosos investigadores en las áreas de la salud y las ciencias sociales, orientan sus esfuerzos para caracterizar el entorno físico y social que se presenta en los procesos de discapacidad y por tal razón se describe que existe una conversión que involucró el paso del estudio médico a uno con orientación social (Oliver, 1990). El caso del Instituto de educación especial COMFABOY en Tunja- Colombia, no es ajeno a esta situación, los siguientes resultados, permitieron caracterizar la calidad de vida en dos pacientes, a través de la evaluación con la escala INICO- FEAPS adaptada y validada en la población colombiana.

Tabla I. A. Resumen puntajes directos autoinforme para las dimensiones de calidad de vida escala INICO-FEAPS (AFFM) B. Perfil de calidad de vida autoinforme escala INICO-FEAPS (AFFM) 


\begin{tabular}{|c|c|c|c|}
\hline \multicolumn{4}{|c|}{ 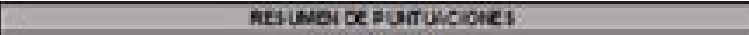 } \\
\hline \multicolumn{4}{|c|}{ UITINGCRS } \\
\hline $\begin{array}{l}\text { onevodics de la } \\
\text { eNrmo oc wha }\end{array}$ & 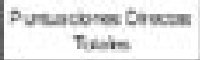 & $\begin{array}{l}\text { Pususiores } \\
\text { Rativele }\end{array}$ & $\begin{array}{l}\text { Aocorsl of ins } \\
\text { Nimentive }\end{array}$ \\
\hline LTCOCTHMECOW & 3 & 18 & n \\
\hline Dcetoids & 2 & $n$ & 30 \\
\hline AOCSTMR:CLOOONW & x & ne & 헤 \\
\hline Renisoiscal & $\bar{x}$ & 9 & F \\
\hline 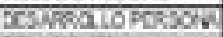 & 8 & 75 & 36 \\
\hline 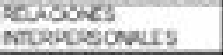 & so & 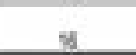 & 8. \\
\hline DGE SIVI WASARK & 3 & qu & 34 \\
\hline DicR STAR RBOOO & $x$ & 8 & ic \\
\hline \multicolumn{2}{|c|}{ 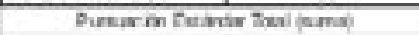 } & \multicolumn{2}{|c|}{$\overline{f o n}$} \\
\hline \multicolumn{2}{|c|}{ Qumponde } & \multicolumn{2}{|c|}{ tad } \\
\hline \multicolumn{3}{|c|}{ 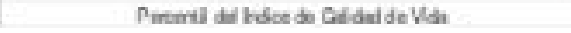 } & $a$ \\
\hline
\end{tabular}

La tabla I muestra el resumen de las puntuaciones estándar en el paciente AFFM (A), en el anterior se pudo evidenciar que las dimensiones de calidad de vida se encuentran en un rango de 9 a 16 lo que se describe de igual forma, en el perfil de calidad de vida (B). El autoinforme y el perfil de calidad de

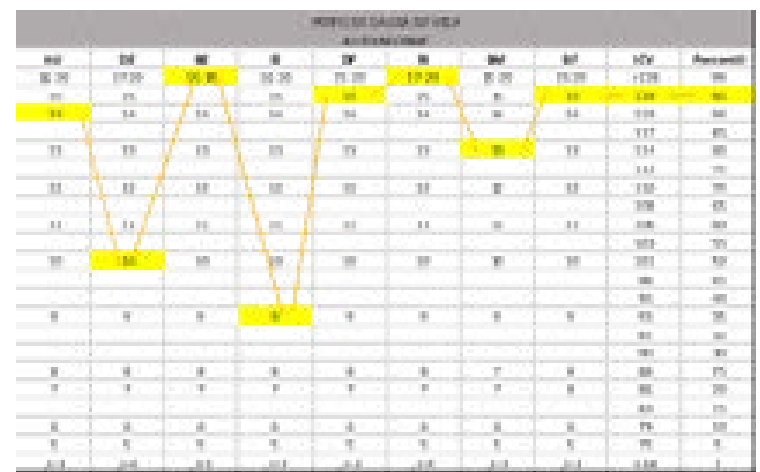

vida muestran además que la mayor puntuación se obtuvo en la dimensión de relaciones interpersonales; por otra parte, la menor puntuación se encuentra en la dimensión de inclusión social.

Tabla 2. A. Resumen puntajes directos informe de otras personas para las dimensiones de calidad de vida escala INICOFEAPS (AFFM) B. Perfil de calidad de vida informe de otras personas escala INICO-FEAPS (AFFM)

\begin{tabular}{|c|c|c|c|}
\hline \multicolumn{4}{|c|}{ 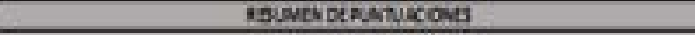 } \\
\hline \multicolumn{4}{|c|}{ 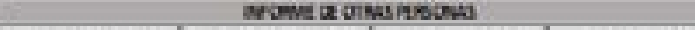 } \\
\hline $\begin{array}{l}\text { busisonestia } \\
\text { cupoupevion }\end{array}$ & $\begin{array}{c}\text { hrtacones Drasas } \\
\text { poste }\end{array}$ & $\begin{array}{l}\text { Rortacoves } \\
\text { esnar }\end{array}$ & $\begin{array}{l}\text { neard be las } \\
\text { Denterst }\end{array}$ \\
\hline 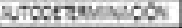 & II & 2 & 7 \\
\hline 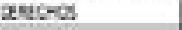 & 19 & $z$ & $\mathbf{z}$ \\
\hline Mverin morosu, & 23 & 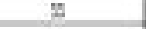 & a \\
\hline MCUBON $500 \mathrm{~s}$ & at & 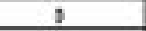 & 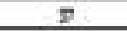 \\
\hline 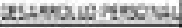 & 2. & 2 & 8 \\
\hline $\begin{array}{l}\text { euxioves } \\
\text { Anruspersan }\end{array}$ & $n$ & 2 & $\pi$ \\
\hline 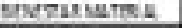 & $\mathrm{x}$ & 2 & $\pi$ \\
\hline Evestaknisco & $2 z$ & $\mathrm{a}$ & 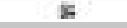 \\
\hline \multicolumn{2}{|c|}{ 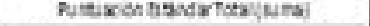 } & 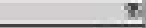 & \\
\hline \multicolumn{2}{|c|}{ 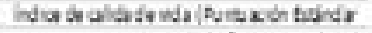 } & 19 & \\
\hline
\end{tabular}

La tabla 2 muestra el resumen de las puntuaciones estándar del informe de otras personas en el paciente AFFM (A), donde se pudo evidenciar que las dimensiones de calidad de vida se encuentran en un rango de 9 a 14 lo que se describe de igual forma, en el perfil de calidad de vida (B). El informe de otras personas y el perfil de calidad de vida muestran además que la

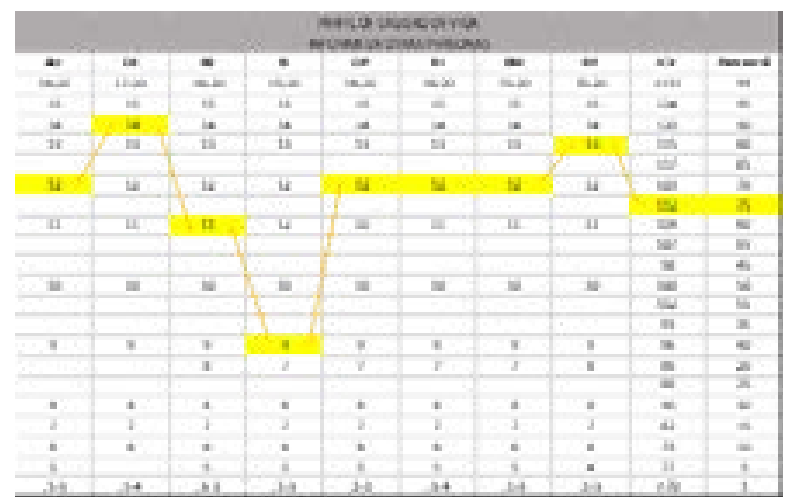

menor puntuación se encuentra en la dimensión de inclusión social; por otra parte, la mayor puntuación se obtuvo en la dimensión de derechos. Es importante resaltar que el Índice de calidad de vida (ICV) en los dos tipos de informe obtuvo una puntuación sobre 100.

Tabla 3. A. Resumen puntajes directos autoinforme para las dimensiones de calidad de vida escala INICO-FEAPS (ORHM) B. Perfil de calidad de vida autoinforme escala INICO-FEAPS (ORHM)

\begin{tabular}{|c|c|c|c|}
\hline \multicolumn{4}{|c|}{ REAmen deruntusookes } \\
\hline \multicolumn{4}{|c|}{ Avoverat } \\
\hline 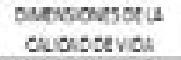 & 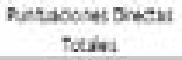 & 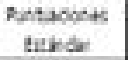 & $\begin{array}{l}\text { Amontistat } \\
\text { Derstuores }\end{array}$ \\
\hline ercominawes & m & st & 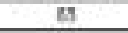 \\
\hline Xutorcs & $\equiv$ & 7 & 28 \\
\hline ร:VEบF:20.0NA & 2 & 重 & x \\
\hline acouson socas & $z$ & 2 & $\mathbf{s}$ \\
\hline 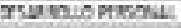 & I & I1 & 3 \\
\hline exours & & & \\
\hline Aropgonones & 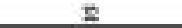 & se & $n$ \\
\hline Sbetamise & 5 & $2 \pi$ & $n$ \\
\hline 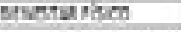 & m & 51 & a \\
\hline \multicolumn{2}{|c|}{ 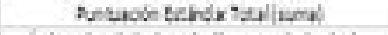 } & \multicolumn{2}{|l|}{ 透 } \\
\hline \multicolumn{2}{|c|}{ 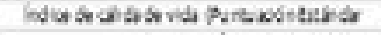 } & \multirow[t]{2}{*}{121} & \\
\hline netar & 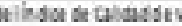 & & \\
\hline
\end{tabular}

La tabla 3 muestra el resumen de las puntuaciones estándar del autoinforme en el paciente ORHM (A), donde se pudo evidenciar que las dimensiones de calidad de vida se encuentran en un rango de 7 a 15 lo que se describe de igual forma, en el perfil de calidad de vida (B). El autoinforme y el perfil de

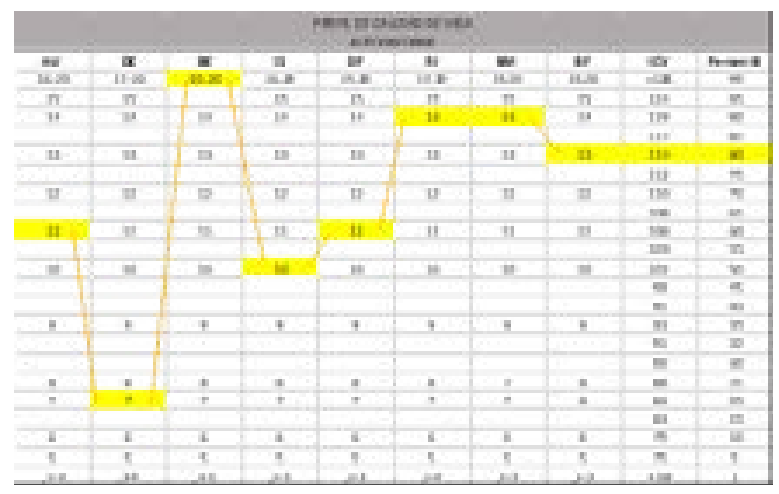

calidad de vida muestran además que la menor puntuación se encuentra en la dimensión de derechos; por otra parte, la mayor puntuación se obtuvo en la dimensión de bienestar emocional. 
Tabla 4. A. Resumen puntajes directos informe de otras personas para las dimensiones de calidad de vida escala INICOFEAPS (ORHM) B. Perfil de calidad de vida informe de otras personas escala INICO-FEAPS (ORHM)

\begin{tabular}{|c|c|c|c|}
\hline \multicolumn{4}{|c|}{ 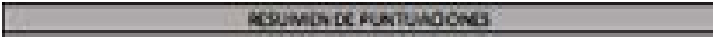 } \\
\hline \multicolumn{4}{|c|}{ insolwit ac oines ressoms } \\
\hline 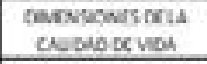 & 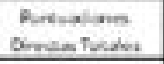 & $\begin{array}{l}\text { Puniwiones } \\
\text { Colbete }\end{array}$ & $\begin{array}{l}\text { Anewils in } \\
\text { Oresives }\end{array}$ \\
\hline 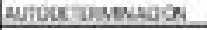 & 8 & 20 & 糧 \\
\hline DOMOOS & 2) & $2 \mathbb{2}$ & sin \\
\hline GUESIARLOOOONAS & $y$ & 13 & is \\
\hline 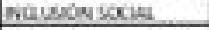 & 31 & 12 & 8 \\
\hline gusupsiprosonas & 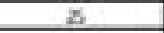 & 2 & $3 z$ \\
\hline RLECTONSS & & & \\
\hline moreposonines & y & 12 & $\pi$ \\
\hline 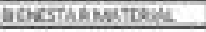 & in & Ii & S1 \\
\hline aUESARIBOCO & Af & ja & 量 \\
\hline \multicolumn{2}{|c|}{ 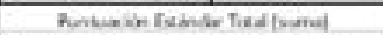 } & is & \\
\hline \multicolumn{2}{|c|}{ 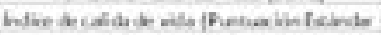 } & 13 & \\
\hline hemets & 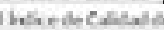 & & \\
\hline
\end{tabular}

La tabla 4 muestra el resumen de las puntuaciones estándar del informe de otras personas en el paciente ORHM (A), donde se pudo evidenciar que las dimensiones de calidad de vida se encuentran en un rango de 9 a 14 lo que se describe de igual forma, en el perfil de calidad de vida (B). El informe de otras personas y el perfil de calidad de vida muestran además que la

\section{DISCUSIÓN Y CONCLUSIONES}

La presente investigación se desarrolló teniendo en cuenta la validación de la escala INICO- FEAPS adaptada a la población colombiana (Henao-Lema, Verdugo- Alonso, CórdobaAndrade, 2015), la cual en el caso del instituto COMFABOY en Tunja- Colombia, demostró tener un enfoque apropiado para evaluar a los pacientes en las diferentes dimensiones. Lo anterior teniendo en cuenta que la calidad de vida se genera a partir de la transformación, construcción social y el mejoramiento de organizaciones que proporcionan servicios de salud, sociales y educativos (Verdugo Alonso et al. 20I3).

En el análisis por dimensiones de la escala de calidad de vida, se destaca que las 2 personas evaluadas presentaron diversas puntuaciones, tanto en el autoinforme como en el informe de otras personas. No obstante, se destaca que el evaluado AFFM presenta puntuaciones similares en el autoinforme e informe de otros, para las dimensiones de bienestar material e inclusión social, evidenciando que la persona en condición de discapacidad se siente a gusto con su situación financiera y sus posesiones coincidiendo con la percepción familiar. Por lo anterior es importante resaltar que el estatus económico favorece la calidad de vida de pacientes en condición de discapacidad y puede favorecer procesos de integración y participación en la comunidad que mejoran la calidad de vida. Esto coincide con lo reportado por Victoria (20/3), quien define que los valores que fundamentan los derechos humanos, son la igualdad y la dignidad humana y con ellos se propician cambios que disminuyen las barreras sociales $y$ dan lugar a la inclusión social de los individuos pese a la diversidad. Por otra parte, las evaluaciones del autoinforme y el informe de otras personas para AFFM en la dimensión de derechos no coincide, mostrando que los dos escenarios se pueden ver afectados por la falta de igualdad, y posiblemente se ven vulnerados algunos derechos que disminuyen la calidad de vida según la percepción del individuo en condición de discapacidad. Lo anterior también ha sido revisado por Flores (20I0), quien menciona que existe un reto frente a la organización de sociedades que integren e impartan justicia

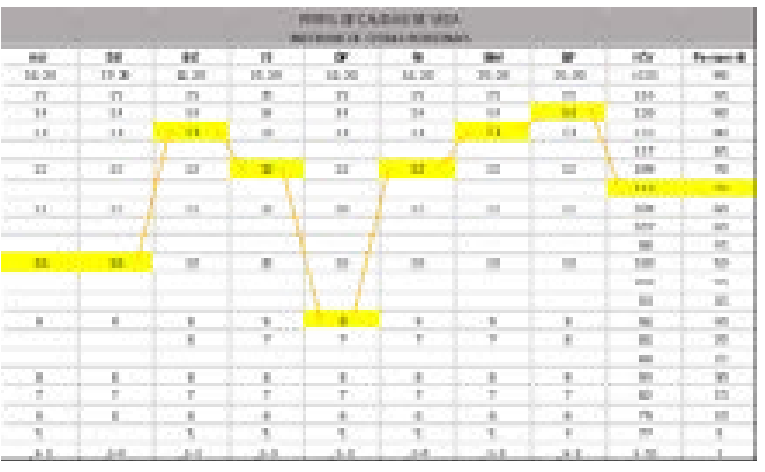

menor puntuación se encuentra en la dimensión de desarrollo personal; por otra parte, la mayor puntuación se obtuvo en la dimensión de bienestar físico. En este paciente evaluado el Índice de calidad de vida (ICV) en los dos tipos de informe obtuvo una puntuación sobre 100.

para hacer efectivos los principios de no discriminación sobre la población discapacitada.

El caso del paciente ORHM, muestra a las dimensiones de bienestar físico y bienestar emocional con una percepción positiva similar en el autoinforme y el informe de otros; lo anterior coincide con lo reportado por Córdoba L, Henao $\mathrm{CP}$, Verdugo MA (2015) quienes mencionan que la escasez de la actividad física de la población adulta en condición de discapacidad, puede generar situaciones de preocupación que van en detrimento de la calidad de vida. No obstante, en el caso de ORHM esta dimensión aporta al mejoramiento de la calidad de vida debido a la adecuada condición nutricional, las posibilidades de entretenimiento y ocio que brinda la familia al individuo en condición de discapacidad y con ello el respaldo y la seguridad que se evidencia en el entorno familiar (Cuervo Rodríguez, 2014).

Por su parte la dimensión de derechos no es percibida de forma similar en el autoinforme y el informe de otros en el caso de ORHM, evidenciando que tanto el individuo como su familia, ven afectada su calidad de vida, posiblemente por la falta de igualdad en diversos aspectos. Lo anterior se puede mejorar teniendo en cuenta que las políticas actuales, se han encauzado para construir acciones que faciliten la organización de sociedades más participativas, incluyentes y respetuosas de los derechos humanos (Victoria, 20I3).

- $\quad$ El diseño de la tarjeta con iconos representativos de las opciones de respuesta del autoinforme, presenta algunas dificultades frente a la evaluación de pacientes con limitaciones en comprensión lectora y comunicación. No obstante, la escala INICO-FEAPS es una herramienta adecuada para evaluar personas en condición de discapacidad intelectual.

- $\quad$ En los pacientes evaluados, los menores puntajes en las dimensiones de inclusión social y derechos, muestran la necesidad de orientar a las familias en procesos que mejoren la compresión de sus derechos y que estos le permitan ser parte 
de una sociedad incluyente.

- $\quad$ El propósito del investigador es que, con el manejo acertado de los resultados obtenidos en la aplicación de la escala, se puedan entregar a cada familia algunas observaciones, para que sean empleadas en el mantenimiento y desarrollo de aquellas dimensiones que se evidenciaron como deficitarias.

- $\quad$ Se pretende que en próximos estudios se logre aplicar la escala INICO-FEAPS en una población más extensa, de esta forma el análisis de los resultados determinará la calidad de vida de una porción importante de la población en condición de discapacidad del municipio de Tunja- Colombia.

- Se ha confirmado a través del proceso de investigación lo que en un principio se formuló como hipótesis y es que la calidad de vida en personas con discapacidad intelectual establecida con la escala INCO-FEAPS es un paso fundamental para mejorar el desarrollo personal y la inclusión social de la población objeto del estudio, además se espera que con las sugerencias entregadas a cada familia la evolución pueda ser lograda en el menor tiempo posible. 


\section{Referencias bibliográficas}

Cobo Mejia, E., Sandoval Cuellar, C \& Alvarado Rojas,

Y. (2012). Magnitud de la discapacidad en Boyacá, Colombia. Salud

Pública, I4(5), 776-788.

Córdoba, L., Mora, A., Bedoya, Á., \& Verdugo, M. (2007). Familias de Adultos con Discapacidad Intelectual en Cali, Colombia, Desde el Modelo de Calidad de Vida. Psykhe (Santiago), 16(2), 29-42. http://dx.doi.org/I0.4067/s07/8-22282007000200003

Cuervo Rodriguez, T. (2014). calidad de vida y

necesidades de apoyo. Uso y análisis de las escalas inico-feaps y sis.

Universidad de Oviedo.

Flores Salgado, L. (2010). Las personas discapacitadas como grupo vulnerable a la luz de la Constitución mexicana.IUS. Revista del Instituto de Ciencias Jurídicas de Puebla A.C., IV (26), I I3-I25. <http://www.redalyc.org/articulo.oa?id=293222980007>

Henao Lema, C., Verdugo Alonso, M \& Córdoba Andrade, L. (2017). Adaptación para Colombia de la Escala INICO-FEAPS de evaluación de calidad de vida de personas con discapacidad intelectual. Retrieved 24 October 2017.

Higuita-Gutiérrez, L., \& Cardona-Arias, J. (2016). Calidad de vida de adolescentes escolarizados de Medellín-Colombia. Revista Facultad Nacional De Salud Pública, 34(2), I45-I55. http://dx.doi.org/I0.17533/udea.rfnsp.v34n2a03

Martínez-Rozo, A. M., Uribe-Rodríguez, A. F \& Velázquez-González, H. J. (2015). La discapacidad y su estado actual en la legislación colombiana. Duazary, I2(I), 49-58.

Mirón Canelo JA, Alonso Sardón M., Serrano López de las Hazas A., Sáenz González MC. (2008) Calidad de vida relacionada con la salud en personas con discapacidad intelectual en España. Rev Panam Salud Publica. 24(5), 336-44.

Muñoz Valdés, Y., Poblete Toloza, Y \& Jiménez Figueroa, E. (2012). Calidad de vida familiar y bienestar subjetivo en jóvenes con discapacidad intelectual de un establecimiento con educación especial y laboral de la ciudad de talca. Interdisciplinaria, 29(2), 207221 .

Nussbaum, M. C., Sen, A \& Reyes Mazzoni, R. R. (Eds.). (1996). La Calidad De Vida. México: Fondo de cultura económica.

Oliver M. The politics of disablement. Basingstoke, Macmillan and St Martin's Press, 1990

Osorio Alcalde, J.C. (2016). La calidad de vida. Revista Eleuthera, 14,

I29-|32. DOI: I0.17|5I/eleu.2016.14.9.

Otzen, Tamara \& Manterola, Carlos. (20 I7). Técnicas de Muestreo sobre una Población a Estudio. International Journal of Morphology, 35(I), 227-232. https://dx.doi.org/10.4067/S07I7-950220I7000100037

Schalock RL, Verdugo MA. Calidad de vida: manual para profesionales de la educación, salud y servicios sociales. Madrid: Alianza Editorial; 2003.

Schalock, R. L., Borthwick-Duffy, S. A., Bradley, V. J., Buntinx, W. H. E., Coulter, D. L., Craig, E. M., ... Yeager, M. H. (20I0). Intellectual Disability: Definition, Classification, and Systems of Supports. Eleventh Edition. American Association on Intellectual and Developmental Disabilities.

Torres Navarro, M. (20I3). Recursos metodológicos en educación física con alumnos con discapacidad física y psíquica. Alpedrete, Madrid: Pila Teleña.

Verdugo M., Gómez L., Arias B., Santamaría M., Clavero D., Tamarit J. (20I3). Evaluación de la calidad de vida en personas con discapacidades intelectuales o del desarrollo: la escala INICIO-FEAPS. Siglo Cero. Revista Española sobre Discapacidad Intelectual, 44(3), 247: 6-20.

Victoria Maldonado, Jorge A. (2013). El modelo social de la discapacidad: una cuestión de derechos humanos. Boletín mexicano de derecho comparado, 46(I38), I093-I I09. http://www.scielo.org.mx/scielo.php?script=sci_arttext\&pid=S004 I$863320 \mid 3000300008 \& \operatorname{lng}=e s \&$ tlng $=e s$. 
Figueredo Vila, E. R. efigueredov@udg. co. c., Campuzano Peña, R. rcampuzanop@udg. co. c., \& Rodríguez Vázquez, C. M. crodriguezv@udg.co.c. (2019). Estrategia compensatoria dirigida a la estimulación del pensamiento en escolares con discapacidad intelectual leve. (Spanish). Dilemas Contemporáneos: Educación, Política y Valores, 6(2), I-20. Retrieved from http://search. ebscohost.com/login.aspx?direct $=$ true\&db=eue\&AN = 134365732\&site=ehost-live

Cuesta-Gomez, J. L., Vidriales-Fernandez, R., \& Carvajal-Molina, F. (2016). [Quality of life in children and adolescents with autism spectrum disorder without intellectual disability]. Revista De Neurologia, 62 Suppl I, S33-S39. Retrieved from http://search. ebscohost.com/login.aspx?direct $=$ true\&db $=$ mdc\&AN $=26922957 \&$ site $=$ ehost-live

Valarezo Mendoza, E. V., Bayas Cano, A. G., Aguilar Chasipanta, W. G., Paredes Navarrete, L. R., Paucar Ipiales, E. N., \& Romero Frómeta, E. (20I7). Programa de actividades físico-recreativas para desarrollar habilidades motrices en personas con discapacidad intelectual. Revista Cubana de Investigaciones Biomédicas, 36(I), I-13. Retrieved from http://search.ebscohost.com/login.aspx?dire $\mathrm{ct}=$ true\&db $=$ asn\&AN $=126336636 \&$ lang $=$ es\&site $=$ ehost-live

Katz, G., \& Lazcano-Ponce, E. (2008). Intellectual disability: definition, etiological factors, classification, diagnosis, treatment and prognosis. Salud Pública de México, 50(2), SI32-SI4I. Retrieved from http://search.ebscohost.com/login.aspx?direct $=$ true\&db=bt h\&AN $=317 \mid 5659 \&$ lang $=$ es\&site $=$ ehost-live

Reyes Izaguirre, J. L. jorgeri@dpe. It. rimed. c. (2017). La inclusión educativa de los escolares con discapacidad intelectual en la educación primaria. (Spanish). Dilemas Contemporáneos: Educación, Política y Valores, 4(3), I-14. Retrieved from http://search. ebscohost.com/login.aspx?direct $=$ true \&db=eue\&AN = |2536 | |57\&lang=es\&site=ehost-live

MUÑOZ VALDÉS, Y. A., POBLETE TOLOZA, Y. D. P., \& JIMÉNEZ FIGUEROA, A. E. (2012). Calidad De Vida Familiar Y Bienestar Subjetivo en Jóvenes Con Discapacidad Intelectual De Un Establecimiento Con Educación Especial Y Laboral De La Ciudad De Talca. Interdisciplinaria: Revista de Psicología y Ciencias Afines, 29(2), 207-22I. Retrieved from http://search.ebscohost.com/login.aspx?di rect $=$ true \&db $=$ asn\&AN $=87912$ I $06 \&$ lang $=$ es\&site $=$ ehost-live

Zapata, A., Bastida, M., Quiroga, A., Charra, S., \& Manuel Leiva, J. (2013). Evaluación Del Bienestar Psicológico Y Estrategias De Afrontamiento en Padres Con Niños O Adolescentes Con Retraso Mental Leve. Psiencia: Revista Latinoamericana de Ciencia Psicológica, 5(I), 15-23. https://doi.org//0.5872/psiencia/5.I.22

Lima-Rodríguez, J. S., Baena-Ariza, M. T., Domínguez-Sánchez, I., \& Lima-Serrano, M. (2018). Intellectual disability in children and teenagers: Influence on family and family health. Systematic review. Enfermeria Clinica, 28(2), 89-102. https://doi.org//0.1016/j. enfcli.20I7.10.005

Huaiquián Billeke, C., Arriagada Vega, C., Betanzo Briones, A., Inostroza Soto, H., \& Llanquitruf Paillán, K. (2018). Manifestaciones afectivas en jóvenes con discapacidad intelectual. Interdisciplinaria: Revista de Psicología y Ciencias Afines, 35(I), 69-85. Retrieved from http://search.ebscohost.com/login.aspx?direct =true\&db=asn\&AN = 322333 I $\&$ \&lang =es\&site =ehost-live

Frielink, N., Schuengel, C., \& Embregts, P. J. C. M. (2018). Autonomy Support, Need Satisfaction, and Motivation for Support Among Adults With Intellectual Disability: Testing a Self-Determination Theory Model. American Journal on Intellectual \& Developmental Disabilities, I23(I), 33-49. https://doi.org/10.1352/1944-7558-123.1.33

VEGA CÓRDOVA, V., JENARO RÍO, C., FLORES ROBAINA, N., CRUZ ORITZ, M., \& LERDO DE TEJADA, A. (20I3). Evaluación de la calidad de vida de adultos con discapacidad intelectual institucionalizados en Chile. Universitas Psychologica, I2(2), 47I-48I. https://doi.org/I0. I I 144/Javeriana.UPSYI2-2.ecva

del Álamo Martín, M. T., \& Ruiz Sanz, M. (2017). Análisis de la calidad de vida de las personas con discapacidad intelectual: un estudio comparativo entre gitanos y no gitanos. Cuadernos de Trabajo Social, 30(2), 45 I-46I. https://doi.org/I0.5209/CUTS.52I8I

Pallisera Díaz, M., Fullana Noell, J., Puyaltó Rovira, C., Vilà Suñé, M., Valls Gabernet, M. J., Díaz Garolera, G., \& Castro Belmonte, M. (2018). Retos para la vida independiente de las personas con discapacidad intelectual. Un estudio basado en sus opiniones, las de sus familias y las de los profesionales. Spanish Journal of Disability Studies / Revista Española de Discapacidad, 6(I), 7-29. https://doi. org/l0.5569/23405104.06.01.01

Verdugo, M. A., Vicente, E., Fernández-Pulido, R., Gómez-Vela, M., Wehmeyer, M. L., \& Guillén, V. M. (20I5). A psychometric evaluation of the ARC-INICO Self-Determination Scale for adolescents with intellectual disabilities. International journal of Clinical Health \& Psychology, I5(2), 149-159. https://doi.org/10.1016/j.ijchp.2015.03.00I

Boluarte Carbajal, A., \& Tamari, K. (2017). Validez de contenido y confiabilidad inter-observadores de Escala Integral Calidad de Vida. Psicología (02549247), 35(2), 64I-666. https://doi.org/I0.18800/psico.20I702.009 
Zorrilla, I. H., Verdugo Alonso, M. Á., Gómez Sánchez, L. E., Ezquerra, S. F., \& Fernández, P. C. (20I5). Evaluación de la calidad de vida en personas con discapacidades significativas: aplicación de la Escala San Martín en la Fundación Obra San Martín. Spanish Journal of Disability Studies / Revista Española de Discapacidad, 3(I), 93-105. https://doi.org//0.5569/2340-5I04.03.0I.05

Pallisera Díaz, M., Fullana Noell, J., Rovira, C. P., Vilà Suñé, M., \& Díaz Garolera, G. (2017). Apoyando la participación real de las personas con discapacidad intelectual: una experiencia de investigación inclusiva sobre vida independiente. Spanish Journal of Disability Studies / Revista Española de Discapacidad, 5(I), 7-24. https://doi.org/I0.5569/2340-5 I04.05.0I.0I

Llamazares de Prado, J. E. luismi-mi@hotmail. e., Arias Gago, A. R. ana. arias@unileon. e., \& Melcon Alvarez, M. A. mmela@ unileon. e. (2017). Calidad de vida, Educación y Ámbito Familiar en la Discapacidad Visual desde la Revisión Teórica. (Spanish). Revista Eletrônica Em Gestão, Educação e Tecnologia Ambiental, 2I (I), 62-77. https://doi.org/10.5902/2236I I 7025699

Ana María, R. C. (20I5). Evaluación de la calidad de vida escolar de personas con discapacidad en FES Zaragoza-UNAM. Revista Mexicana de Orientación Educativa, 12(29), 18-25. Retrieved from http://search.ebscohost.com/login.aspx?direct=true\&db=asn\& AN $=|| 3 \mid 25383 \&$ lang $=$ es\&site $=$ ehost-live

Alba Viviana Herrera, H. (2015). Narrativas de Madres: acerca de las concepciones y prácticas del cuidado en la primera infancia con discapacidad y su incidencia en la calidad de vida en la localidad de San Cristóbal. Revista CIFE, I7(26), 109-I48. Retrieved from http://search.ebscohost.com/login.aspx?direct = true\&db=asn\&AN = I 19215006\&lang=es\&site= ehost-live

Castro, Lilian, Vallejos, Viviana, Casas, José A., Cerda, Gamal, Sánchez, Sergio, \& Zuñiga, Daniela. (2017). Adaptación de la Escala Integral de Calidad de Vida en personas con discapacidad intelectual y del desarrollo en población chilena. Terapia psicológica, 35(3), 23I-238. https://dx.doi.org//0.4067/S07/8-480820I700030023 I

Córdoba Andrade, Leonor, Henao Lema, Claudia Patricia, \& Verdugo Alonso, Miguel Ángel. (2016). CALIDAD DE VIDA DE ADULTOS COLOMBIANOS CON DISCAPACIDAD INTELECTUAL. Hacia la Promoción de la Salud, 2 I (I), 91 - I05. https://dx.doi. org/10.17151/hpsal.2016.21.1.8

Henao-Lema, Claudia Patricia, Verdugo-Alonso, Miguel Ángel, \& Córdoba-Andrade, Leonor. (2015). Adaptación para Colombia de la Escala INICO-FEAPS de evaluación de calidad de vida de personas con discapacidad intelectual. Revista de la Facultad de Medicina, 63(4), 677-686. https://dx.doi.org/10.15446/revfacmed.v63.n4.50799

Gil Obando, L., López López, A., Barreiro Novoa, S., Molina Heredia, Y., \& Solano Esparragoza, Z. del. (2018). Discapacidad y calidad de vida en población adulta del Municipio de Soledad, Atlántico - Colombia. Inclusión \& Desarrollo, 5(2), I43-I58. https:// doi.org/I0.26620/uniminuto.inclusion.5.2.2018.143-158 